\title{
Adsorption and Desorption Characteristics of Nanozeolites as Adsorbent for Dimethyl Methylphosphonate
}

\author{
Xinming $\mathrm{Ji}^{*}$, Wei Yao, Yuanyuan $\mathrm{Hu}^{1}$, Nan Ren ${ }^{1}$, \\ Jia Zhou, Yiping Huang and Yi Tang ${ }^{1}$ \\ ASIC and System State Key Lab, Department of Microelectronics, \\ Fudan University, Shanghai 200433, P. R. China \\ ${ }^{1}$ Department of Chemistry, Fudan University, Shanghai 200433, P. R. China
}

(Received May 7, 2010; accepted August 13, 2010)

Key words: zeolite, adsorbent, QCM, DMMP

The selective adsorption of dimethyl methylphosphonate (DMMP) onto zeolites is described in this paper. Three types of zeolite, silicalite-1, ZSM (Zeolite Socony Mobil)-5, and Cu-ZSM-5, were synthesized and assembled onto the surface of a quartz crystal microbalance (QCM) covered with a Au film. The QCM was adopted as an effective tool to analyze the adsorption and desorption characteristics. The optimum desorption conditions were determined experimentally as a desorption temperature of $523 \mathrm{~K}$ and a desorption time of $30 \mathrm{~min}$. Good repeatability could be obtained under these optimal desorption conditions. Adsorption capacity was determined by comparing the different zeolites as selective adsorbents for DMMP. Cu-ZSM-5 had the highest adsorption capacity and the best selectivity for the three zeolites. The effect of copper ions on the adsorption of DMMP was tested by synthesizing five Cu-ZSM-5 zeolites with different copper ion contents. Adsorption sensitivity increased in proportion to copper content before saturation. The sensitivity and detection limit of a QCM coated with $\mathrm{Cu}-$ ZSM-5 reached about $8.8 \mathrm{~Hz} / \mathrm{ppm}$ and $0.3 \mathrm{ppm}$, respectively, confirming that $\mathrm{Cu}-\mathrm{ZSM}-5$ zeolite is a promising adsorbent for DMMP detection.

\section{Introduction}

There is increasing interest in the effective detection of chemical warfare agents (CWAs). Owing to safety requirements and government regulations, most work is performed using simulant compounds, such as dimethyl methylphosphonate (DMMP). Detection of trace amounts of analytes from minute quantities of vapors has always been challenging for developing CWA detection systems. To improve the detection limit of sensor systems, a preconcentrator is incorporated into the analytical system to enhance the overall performance of the detector by preconcentrating the analyte using adsorbents. ${ }^{(1-3)}$ Over

*Corresponding author: e-mail: xmji@fudan.edu.cn 
the past several years, compounds in the material class of zeolites have been receiving increasing attention as sensitive adsorbents for gas detection. ${ }^{(4-7)}$

Zeolites are of interest because of their generally high adsorptivities and large surface areas. Combining zeolite adsorbency with potential chemical reactivity has been examined previously in the detection of DMMP. ${ }^{(8,9)}$ The common zeolites tested in the laboratory have shown an outstanding capacity to adsorb DMMP vapor. Xie and coworkers $^{(10,11)}$ studied the ZSM-5 and $\beta$ zeolite applications for DMMP detection in which zeolite films immobilized on a quartz crystal microbalance (QCM) were used as gas sensors. Gas sensors based on nanosized zeolites show a high sensitivity for detecting DMMP. A recent study has focused mainly on the X-type and Y-type zeolites. A number of subsequent studies ${ }^{(9,12,13)}$ of $\mathrm{NaX}$ and $\mathrm{NaY}$ faujasite zeolite chemistry with DMMP have been carried out by Knagge et al. ${ }^{(12)}$ and Yang et al. ${ }^{(13)}$ Their works revealed that DMMP strongly adsorbed to X-type faujasite zeolites and underwent nucleophilic substitution by supercage oxygen atoms.

In several studies, the reaction and decomposition of DMMP on various zeolites have been investigated. However, previous efforts have indicated concerns about the sensitivity of zeolites. In fact, most of these zeolites suffer from high cross-sensitivity to smoke, moisture, $\mathrm{CO}_{2}$ and other interferents in complicated environments, where the competitive adsorption of other constituents lowers the selectivity of $\mathrm{NaX}$ and ZSM-5 zeolites. Therefore, novel sensitive materials should be sought to improve the selectivity of the gas sensor for DMMP detection. The purpose of this study is to assess the capability of DMMP adsorption by a novel selective Cu-ZSM-5 zeolite and to explore how copper species promote the adsorption of volatile DMMP.

In this study, silicalite-1 zeolite was primarily studied because of its stable crystal structure and ease of synthesis. To evaluate the adsorption and desorption characteristics of zeolites, a QCM coated with zeolites was adopted to test the mass of adsorbed/ desorbed DMMP. Previous research demonstrated that $\mathrm{Cu}^{2+}$ could be used to recognize phosphonyl groups owing to the formation of strong $\mathrm{P}=\mathrm{O}-\mathrm{Cu}^{2+}$ bonds. ${ }^{(14,15)}$ Therefore, we modified ZSM-5 zeolite with $\mathrm{Cu}^{2+}$ as a method of increasing adsorption capacity. Five Cu-ZSM-5 zeolites that differed in their $\mathrm{Cu}^{2+}$ ion content were synthesized and analyzed. The relationship between copper content and adsorption response was obtained. A comparison of the characteristics of these different zeolites was also present in this paper.

\section{Experimental Methods}

\subsection{Synthesis and assembly of zeolite films}

\subsubsection{Silicalite-1}

First, the colloidal seed crystals of silicalite-1 were prepared in accordance with the method described by Persson et al. ${ }^{(16)}$ using TPAOH, tetraethoxysilane and distilled water at a ratio of 9 TPAOH: $25 \mathrm{SiO}_{2}: 480 \mathrm{H}_{2} \mathrm{O}: 100$ ethanol. The solution was treated hydrothermally under refluxing conditions at $100^{\circ} \mathrm{C}$ for $48 \mathrm{~h}$. The silicalite- 1 sol was purified several times by centrifugation and redispersion in water until it reached a $\mathrm{pH}$ $<10$. 
The zeolite seeds were then adsorbed onto silane- and polymer-modified gold substrates to assemble a zeolite film on the Au surface of a QCM. ${ }^{(17)}$ Before modifying the organic silane film, the QCM was cleaned twice for $15 \mathrm{~min}$ in an ultrasonic bath, first with acetone and then with distilled water. Silanization of the Au surface was performed in the liquid phase for $3 \mathrm{~h}$ using $10 \mathrm{~m}$ mol $\gamma$-mercaptopropyltrimethoxysilane (MPT). The substrate was immersed in $0.1 \mathrm{~mol}$ of $\mathrm{HCl}$ for $15 \mathrm{~h}$ to hydrolyze the adsorbed MPT, and then rinsed repeatedly with ethanol.

To adsorb a monolayer of silicalite- 1 crystals onto the silane modified Au surface, the QCM was immersed into $30 \mathrm{ml}$ of a sol of colloidal crystals of silicalite- $1 \mathrm{for} 1 \mathrm{~h}$, and then dipped alternately into PDDA and silicalite-1 sol for 20 min to grow a multilayer of silicalite-1 film.

The surface morphology of zeolite films was studied by scanning electron microscopy (SEM). Figure 1(a) clearly shows that the silicalite-1 crystal was successfully deposited on the Au surface of the QCM. The morphology of the synthesized silicalite- 1 crystal was cuboidal and the crystal size was $200 \mathrm{~nm}$, with homogeneous coverage. Ultimately, four layers of a silicalite-1 film were assembled with a final thickness of $800 \mathrm{~nm}$.

\subsubsection{ZSM-5 and Cu-ZSM-5}

The ZSM-5 sol was prepared in accordance with the method described by Persson et al. ${ }^{(18)}$ using TEOS and TPAOH. The solution was treated hydrothermally under reflux condition at $100^{\circ} \mathrm{C}$ for $48 \mathrm{~h}$ to obtain the colloidal seed crystals of ZSM-5.

$\mathrm{Cu}-\mathrm{ZSM}-5$ zeolite was prepared by ion exchange of ZSM-5 and $\mathrm{Cu}\left(\mathrm{NO}_{3}\right)_{2} \cdot{ }^{(19-21)}$ ZSM-5 sol and $\mathrm{Cu}\left(\mathrm{NO}_{3}\right)_{2}$ were mixed together and reacted, followed by 20 min of centrifugation. Unreacted $\mathrm{Cu}\left(\mathrm{NO}_{3}\right)_{2}$ was removed by washing with distilled water. The quantities of $\mathrm{Cu}^{2+}$ exchanged depended on the number of exchanges between ZSM-5 and $\mathrm{Cu}\left(\mathrm{NO}_{3}\right)_{2}$. Five types of $\mathrm{Cu}-\mathrm{ZSM}-5$ zeolite were prepared using the same process by manipulating the number of exchanges.

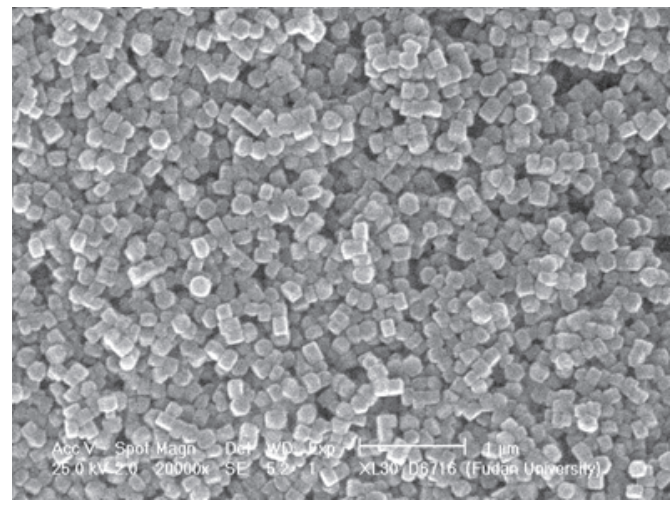

(a)

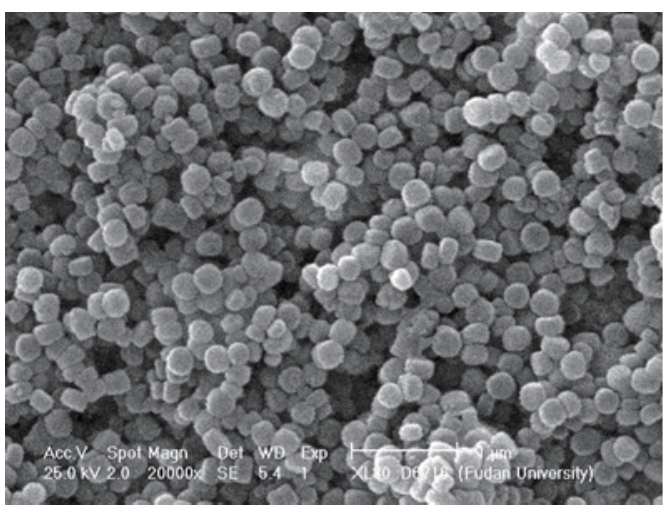

(b)

Fig. 1. SEM images of silicalite-1(a) and Cu-ZSM-5 (b) films on Au substrate. 
The assembly of Cu-ZSM-5 zeolite on the QCM surface was carried out using the same steps as described for silicalite-1. An SEM image of Cu-ZSM-5 with atomic Si/Al ratios of 23 is shown in Fig. 1(b). The crystal size is $250 \mathrm{~nm}$. Three layers are assembled to obtain a thickness of $750 \mathrm{~nm}$. Thus, depositing zeolite films on a Au substrate was feasible by this method.

\subsection{Measurement of $\mathrm{Cu}^{2+}$ content}

The ion-exchanged copper content depended on not only the cation content and $\mathrm{Si} /$ $\mathrm{Al}$ ratio of ZSM-5 zeolite, but also the number of exchanges. The compositions of $\mathrm{Cu}-$ ZSM-5 zeolites were analyzed by energy dispersive X-ray (EDX) spectrometry. Figure 2 shows the EDX spectra of one type of Cu-ZSM-5 zeolite. Table 1 shows the specific $\mathrm{Cu}$ content of five different zeolites: $\mathrm{Cu}-\mathrm{ZSM}-5(1)$ to $\mathrm{Cu}-\mathrm{ZSM}-5(5)$ have copper ion contents of $0.12 \%, 0.39 \%, 1.34 \%, 2.72 \%$, and $4.19 \%$, respectively. These results provide a reliable basis for the following experiments to evaluate the relationship between copper content and adsorption response.

\subsection{Experimental system}

The measurement sensor system is shown in Fig. 3. It includes a sample gas with valves, a flowmeter, the QCM coated with zeolites in a sensing chamber, a frequency meter and a computer. Gas is driven into the chamber through the flowmeter at a constant flow rate. The QCM is then used to test the mass of gas adsorbed by the

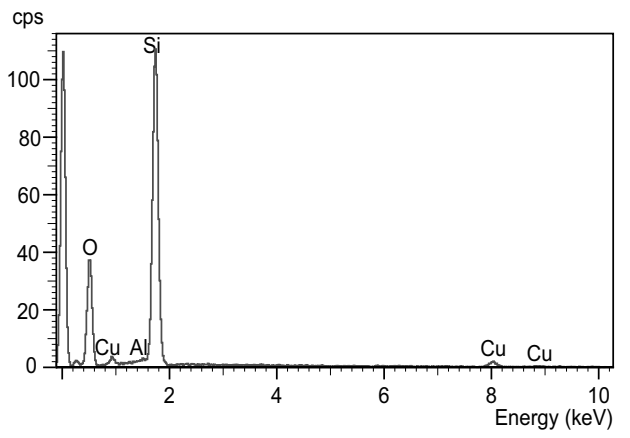

Fig. 2. EDX spectrum of Cu-ZSM-5 zeolite.

Table 1

Composition of $\mathrm{Cu}-\mathrm{ZSM}-5$ zeolites.

\begin{tabular}{ccc}
\hline Zeolite name & Number of exchanges & $\begin{array}{c}\text { Copper content } \\
\text { (molar ratio) }\end{array}$ \\
\hline Cu-ZSM5(1) & 1 & $0.12 \%$ \\
Cu-ZSM5(2) & 2 & $0.39 \%$ \\
Cu-ZSM5(3) & 3 & $1.34 \%$ \\
Cu-ZSM5(4) & 4 & $2.72 \%$ \\
Cu-ZSM5(5) & 5 & $4.19 \%$ \\
\hline
\end{tabular}




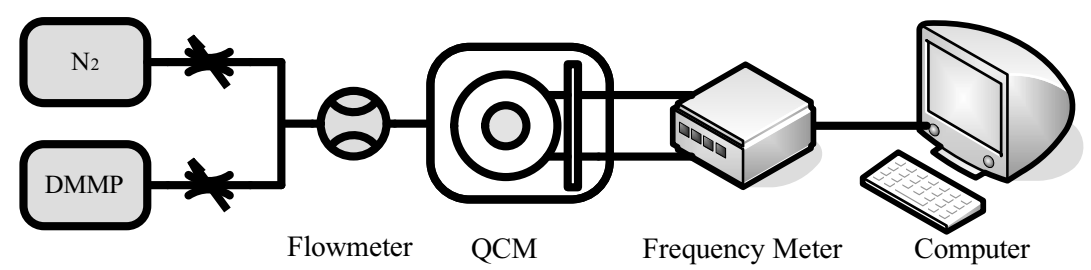

Fig. 3. Schematic of experimental system.

zeolites. The relationship between mass change and frequency shift follows the Sauerbrey equation: ${ }^{(22)}$

$$
\Delta f=-2.26 \times 10^{-6} f^{2} \frac{\Delta m}{A},
$$

where $\Delta f(\mathrm{~Hz})$ is the frequency shift, $f(\mathrm{~Hz})$ is the initial frequency, $\Delta m(\mathrm{~g})$ is the mass change, and $A\left(\mathrm{~cm}^{2}\right)$ is the sensing surface area.

\subsection{Testing}

The working frequency of the QCM was $8 \mathrm{MHz}$. Before the test, nitrogen was driven through the chamber at $100 \mathrm{ml} / \mathrm{min}$ as a background gas until the frequency shift of the QCM controls was within $\pm 2 \mathrm{~Hz}$ in 10 min. Once the QCM reached a balance, the target gas (DMMP) at a certain concentration was driven into the chamber at the same gas flow rate. When the adsorption reached the equilibrium state, the frequency shifts caused by the mass changes due to adsorbed/desorbed gas molecules were automatically recorded using a Chi440 instrument (frequency counter/oscillating circuit). During the test, all the adsorption experiments were conducted at ambient temperature (298 K), while the thermal desorption experiments were operated at different high temperatures controlled by a hotplate.

The adsorption and desorption characteristics of silicalite-1, ZSM-5 and Cu-ZSM-5 zeolite were studied. The adsorption responses to different DMMP concentrations and the selectivity to various gases were tested. In addition, the optimum desorption conditions were characterized. In all the adsorption experiments, the adsorbents were pretreated at $300^{\circ} \mathrm{C}$ for $2 \mathrm{~h}$ to remove organic contaminants and moisture prior to the experiments.

\section{Results and Discussion}

\subsection{Adsorption characterization}

The real-time response of the QCM sensor based on nanosized ZSM-5 zeolite is illustrated in Fig. 4(a). The absorption frequency of the sensor was observed to change immediately as soon as DMMP was injected into the chamber. The response time, defined as the time interval between the injection of the gas and the moment of frequency 


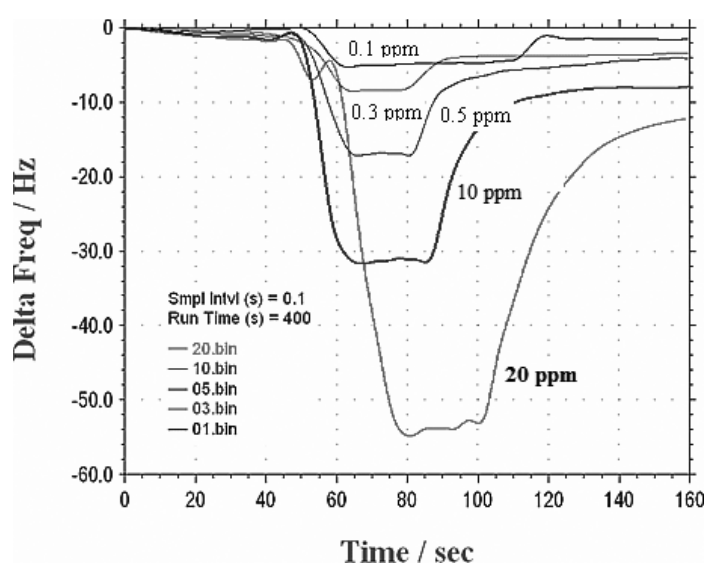

(a)

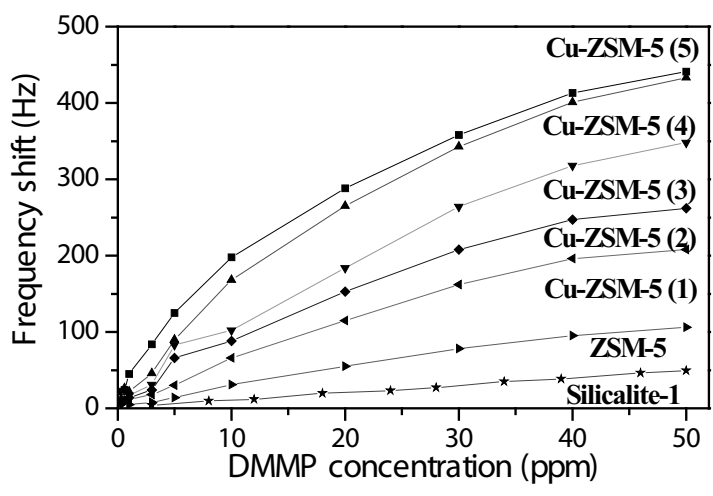

(b)

Fig. 4. (a) Real-time responses of ZSM-5 to different concentrations of DMMP and (b) responses of silicalite-1, ZSM-5 and Cu-ZSM-5 zeolites to different DMMP concentrations.

shift, $(\Delta f)$, reached a maximum of $80 \%$ in less than $100 \mathrm{~s}$. The relationships between frequency shifts and DMMP concentrations are shown in Fig. 4(b) for all zeolites. The data points represent frequency shifts from the equilibrium state in response to DMMP gas. The detection limit of silicalite-1 zeolite was $3 \mathrm{ppm}$ with a sensitivity of $1 \mathrm{~Hz} / \mathrm{ppm}$. Because of the simple physical adsorption, silicalite-1 zeolite showed a good linearity. Compared with silicalite-1, ZSM-5 zeolite showed a higher sensitivity of $2.1 \mathrm{~Hz} / \mathrm{ppm}$ and a lower detection limit of $1 \mathrm{ppm}$. Two reasons could explain this difference. The most significant one is that aluminum species in zeolites play an important role in the adsorption of DMMP molecules. ZSM-5 zeolite contains aluminum species whereas silicalite-1 does not; consequently, ZSM-5 has a better adsorption capacity than silicalite-1. Another factor to be considered is that ZSM-5 has a larger pore size, as shown in Fig. 1, sufficient to allow entry of DMMP molecules into the pores.

All of the Cu-ZSM-5 zeolites exhibited a better capability to adsorb DMMP than ZSM-5 and silicalite-1 zeolites. Cu-ZSM-5 of different $\mathrm{Cu}^{2+}$ concentrations showed completely different detection sensitivities, as shown in Table 2. For the detection of 0.3 ppm DMMP, the sensitivities of different types of Cu-ZSM-5 were $4.16 \mathrm{~Hz} / \mathrm{ppm}, 5.24$ $\mathrm{Hz} / \mathrm{ppm}, 6.9 \mathrm{~Hz} / \mathrm{ppm}, 8.7 \mathrm{~Hz} / \mathrm{ppm}$, and $8.8 \mathrm{~Hz} / \mathrm{ppm}$, with greater adsorption as copper ion content increased. One of the reasons for this increase is that $\mathrm{Cu}^{2+}$ has affinity for DMMP; therefore, more DMMP molecules can be adsorbed by Cu-ZSM-5. A strong bond has been shown to exist between phosphonyls and $\mathrm{Cu}^{2+}$, which combine together to form $\mathrm{Cu}^{2+}-\mathrm{O}=\mathrm{P}$ bonds. ${ }^{(14,15)}$ It is believed that the incorporation of copper species in zeolite can strengthen the electrostatic interaction between DMMP and the adsorbent. According to the results shown in Fig. 4(b), the absorption capacities of the zeolites can be estimated (calculated from Sauerbrey eq. (1)). $1 \mathrm{ml}$ silicalite-1 zeolite can absorb 3.4 
Table 2

Sensitivity of different zeolites to DMMP gas.

\begin{tabular}{cc}
\hline Zeolite & Sensitivity to DMMP gas \\
\hline ZSM-5 & $2.1 \mathrm{~Hz} / \mathrm{ppm}$ \\
$\mathrm{Cu}-Z S M-5(1)$ & $4.1 \mathrm{~Hz} / \mathrm{ppm}$ \\
$\mathrm{Cu}-Z S M-5(2)$ & $5.2 \mathrm{~Hz} / \mathrm{ppm}$ \\
$\mathrm{Cu}-$ ZSM-5(3) & $6.9 \mathrm{~Hz} / \mathrm{ppm}$ \\
$\mathrm{Cu}-Z$ SM-5(4) & $8.7 \mathrm{~Hz} / \mathrm{ppm}$ \\
$\mathrm{Cu}-Z S M-5(5)$ & $8.8 \mathrm{~Hz} / \mathrm{ppm}$ \\
\hline
\end{tabular}

ml DMMP, while $1 \mathrm{ml} \mathrm{Cu}-Z \mathrm{SM}-5(1)$ can absorb $15.6 \mathrm{ml}$ DMMP. This proves that $\mathrm{Cu}-$ ZSM-5 zeolite is a more suitable sensitive adsorbent for DMMP detection.

The relationships between copper content and adsorption capability of the five $\mathrm{Cu}$ ZSM-5 zeolites with different copper contents are presented in Fig. 5. In the range of $0-2.72 \%$, the adsorption response increased linearly with copper content. When the copper content reached $2.72 \%$, the adsorption capacity was saturated. This result suggests that the copper modifier has a limited ability to enhance the sensitivity for the DMMP detection. This may be due to the weakening of the $\mathrm{DMMP}-\mathrm{Cu}^{2+}$ interaction. Although phosphonyl groups have a strong bond with $\mathrm{Cu}^{2+}$ and copper complexes, the overload of copper cation occupies the inherent active sites and blocks the channel of the zeolite to some extent. ${ }^{(21)}$ This would prevent DMMP molecules from entering and diffusing into the zeolite. Consequently, the redundant copper cation contributes less to the absorption of DMMP vapor. Additionally, the incorporation of copper unavoidably reduces the proportion of zeolite in the adsorbent used in the adsorption test.

To test the selectivities of three types of zeolite, their responses to different gases were measured. The adsorptions of $300 \mathrm{ppm} \mathrm{CO}_{2}, 500 \mathrm{ppm} \mathrm{NH}_{3}$ and water vapor by silicalite-1, Cu-ZSM-5(1) and Cu-ZSM-5(5) zeolites are shown in Fig. 6. For both silicalite-1 and Cu-ZSM-5 zeolites, the effect of moisture could not be neglected. Therefore, it is necessary to pretreat the samples to remove moisture prior to the experiments. Silicalite- 1 adsorbs less moisture than Cu-ZSM- 5 because silicalite- 1 is a pure silicon zeolite with hydrophobic properties. For Cu-ZSM-5(5), its response to DMMP is greater than its response to moisture because of its higher copper content. The frequency shifts for $300 \mathrm{ppm} \mathrm{CO}_{2}$ and $500 \mathrm{ppm} \mathrm{NH}_{3}$ are smaller than $50 \mathrm{ppm}$ DMMP, because DMMP is a strong polar molecule that it is easily adsorbed by $\mathrm{Cu}-\mathrm{ZSM}-5$ zeolite. As a result of its polar adsorption, Cu-ZSM-5 zeolite shows a much better selectivity as copper content increased.

\subsection{Desorption characterization}

To use zeolites as preconcentrators in a gas sensor, it is important to evaluate their desorption characteristics and determine their optimum desorption conditions. A high desorption ratio is required for a perfect sorbing material.

The desorption ratio at different temperatures and different times was examined to determine the best desorption condition. DMMP is a strong polar molecule, so it is more difficult for DMMP to desorb than to adsorb. Generally speaking, high temperatures and long times are required to completely desorb DMMP. Figure 7 shows that desorption 


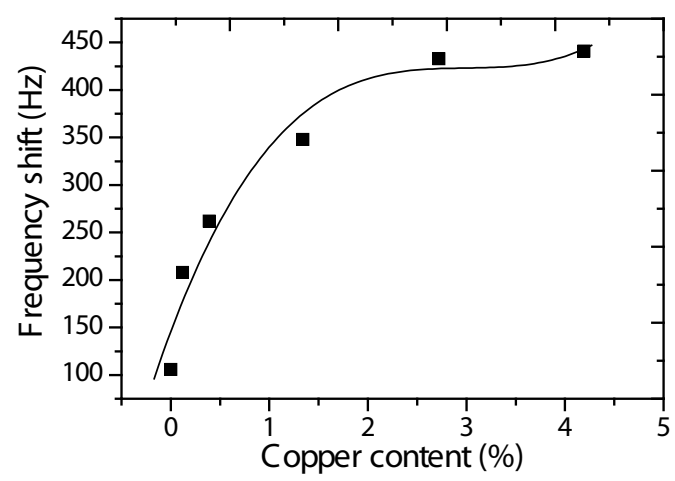

Fig. 5. Relationship between copper content and adsorption response to 50 ppm DMMP.

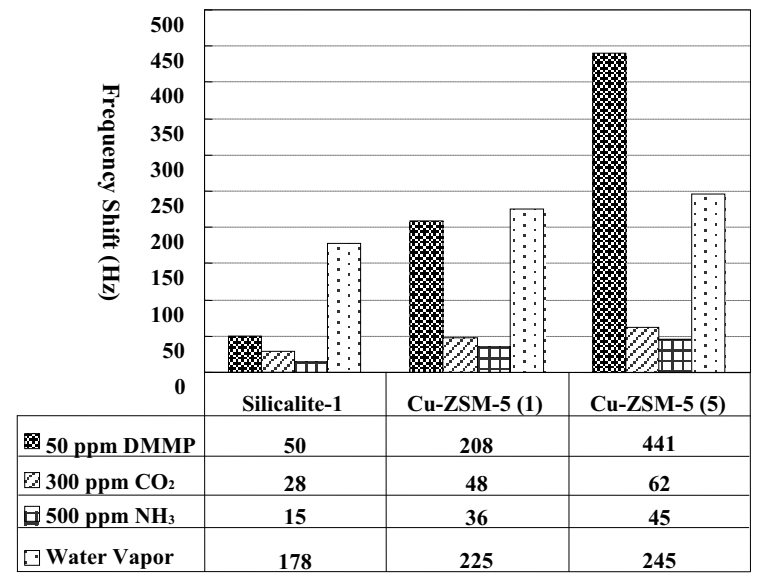

Fig. 6. Response of silicalite-1 and Cu-ZSM-5 zeolites to different gases.

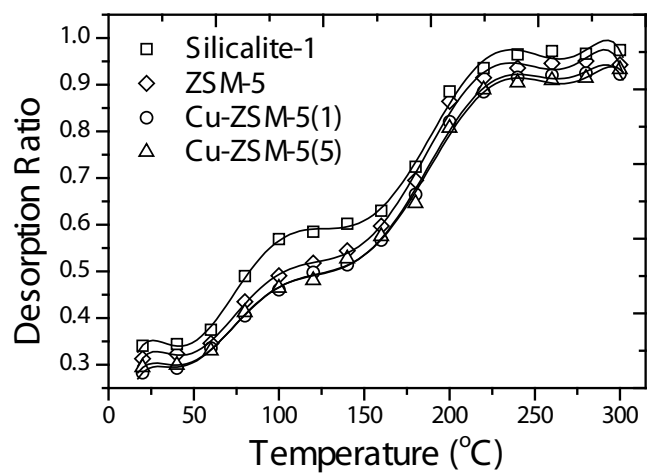

Fig. 7. Relationship between desorption ratio and temperature of silicalite-1, ZSM-5, and CuZSM-5 zeolites. 
ratio increased with temperature. At temperatures above $250^{\circ} \mathrm{C}$, the desorption ratio was maintained at higher than $90 \%$. Cu-ZSM-5 showed a relatively lower desorption ratio than silicalite- 1 and ZSM-5 owing to the affinity between $\mathrm{Cu}^{2+}$ and DMMP. It is difficult to destroy the $\mathrm{Cu}^{2+}-\mathrm{O}=\mathrm{P}$ bonds. Fortunately, the desorption ratio is still sufficiently high for preconcentrator applications. Both Cu-ZSM-5(1) and Cu-ZSM-5(5) zeolites show similar desorption characteristics, which indicates that the copper content has little effect on desorption ratio. Figure 8 shows that it took at least $30 \mathrm{~min}$ for all of the zeolites to achieve a desorption ratio of more than $90 \%$. Therefore, it is obvious that a temperature and time within the flat range of curves should be chosen to obtain a stable and high desorption ratio.

Repetition of adsorption and desorption experiments was performed under the optimal desorption conditions (temperature is $250^{\circ} \mathrm{C}$ and the time of every loop is more than 30 min). Figure 9 shows that there was a good repeatability and more than 90\% DMMP

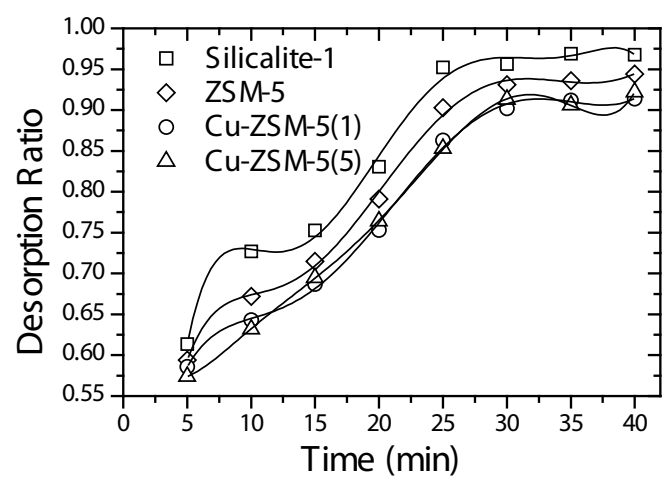

Fig. 8. Relationship between desorption ratio and time of silicalite-1, ZSM-5 and Cu-ZSM-5 zeolites.

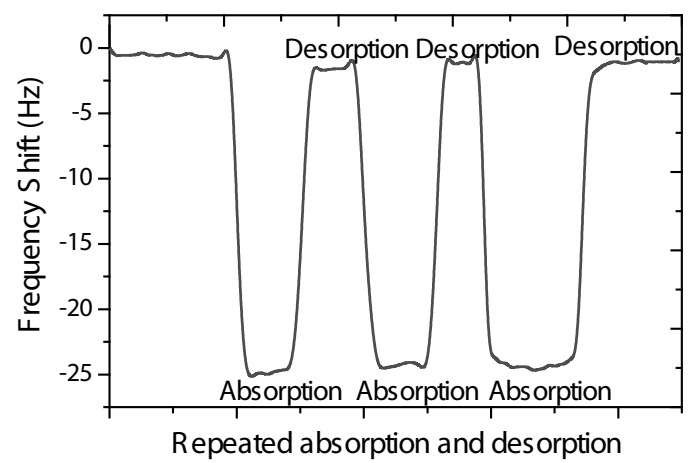

Fig. 9. Repeatability of adsorption and desorption experiments under the optimum conditions. 
was desorbed each time. This constant desorption ratio is essential for calculating the amount of a preconcentrated gas if the zeolites are to be used as preconcentrators.

\section{Conclusions}

In this study, the adsorption and desorption characteristics of nanosized zeolite materials used as an adsorbent in a gas sensor were examined. Silicalite-1, ZSM-5 and Cu-ZSM-5 zeolites were synthesized and deposited on a QCM Au surface. The QCM was chosen as a tool to analyze the properties of the zeolite adsorbents. As demonstrated, Cu-ZSM-5 zeolite showed a higher adsorption capacity owing to the affinity between $\mathrm{Cu}^{2+}$ and DMMP molecules, and its adsorption capacity improved with increasing copper content. However, the copper modifier had a limited ability for ZSM-5 zeolite to enhance the sensitivity and absorption capability for the DMMP detection. When the copper content was more than $2.72 \%$, the adsorption capacity of Cu-ZSM-5 became saturated. Cu-ZSM-5 zeolites also showed fairly good selectivity. Stable and high desorption ratios could be obtained at temperatures higher than $250^{\circ} \mathrm{C}$ and with desorption times longer than $30 \mathrm{~min}$. The results suggest that it is feasible to adopt Cu-ZSM-5 zeolites as adsorbents of DMMP. Further work on combining zeolite preconcentrators with an infrared gas sensor system for DMMP detection is ongoing.

\section{Acknowledgments}

This study was partially supported by the National Science Foundation through Grant No. 90607015 and the Doctoral Fund of the Ministry of Education of China through Grant No. 20070246099.

\section{References}

C. J. Lu and E. T. Zellers: Anal. Chem. 73 (2001) 3449.

2 I. Voiculescu, M. Zaghloul and N. Narasimhan: Trends Anal. Chem. 27 (2008) 327.

3 M. Tagliabue, D. Farrusseng, S. Valencia, S. Aguado and U. Ravon: Chem. Eng. J. 65 (2009) 1.

4 H. Huang, J. Zhou, S. Chen, L. Zeng and Y. Huang: Sens. Actuators, B 101 (2004) 316.

5 E. Alpay, N. Haq, L. S. Kershenbaum and N. F. Kirkby: Gas Sep. Purif. 10 (1996) 25.

6 S. Bhatia, A. Z. Abdullah and C. T. Wong: Applied Catalysis B: Environmental 52 (2004) 91.

7 B. Ledvinkova, F. Keller, J. Kosek and U. Nieken: Chem. Eng. J. 140 (2008) 578.

8 A. Corma: Chem. Rev. 97 (1997) 23.

9 J. B. Sambur, D. C. Doetschman, S.-W. Yang, J. T. Schulte, B. R. Jones and J. B. DeCoste: Microporous Mesoporous Mater. 112 (2008) 116.

10 H. Xie, Qi. Yang and X. Sun: Sens. Mater. 17 (2005) 21.

11 H. Xie, Y. Ting, X. Sun, Z. Jia and Y. Huang: Fifth Inter. Conf. on Thin Film Physics and Applications SPIE. 5774 (2005) 624.

12 K. Knagge, M. Johnson, V. H. Grassian, and S. C. Larsen: Langmuir 22 (2006) 11077.

13 S.-W. Yang, D. C. Doetschman and J. T. Schulte: Microporous Mesoporous Mater. 92 (2006) 56.

14 M. Crooks, H. C. Yang and J. McEllistrem: Faraday Discuss. 107 (1997) 285. 
15 Y. Yang, H.-F. Ji and T. Thundat: J. Am. Chem. Soc. 125 (2003) 1124.

16 A. Persson, B. J. Schoeman, J. Sterte and J. Otterstedt: Zeolites 14 (1994) 557.

17 S. Mintova, V. Valtchev, V. Engstrom, B. J. Schoeman and J. Sterte: Microporous Mater. 11 (1997) 149.

18 A. Persson, B. J. Schoeman and J. Sterte: Zeolites 15 (1995) 611.

19 V. J. Inglezakis, M. D. Loizidou and H. P. Grigoropoulou: Water Res. 36 (2002) 2784.

20 F. N. Gu, T. T. Zhuang, Y. Cao, C. F. Zhou and J. H. Zhu: Solid State Sci. 10 (2008) 1658.

21 E. Broclawik, J. Datka, B. Gil and P. Kozyra: Int. J. Mol. Sci. 3 (2002) 435.

22 G. Z. Sauerbrey: Z. Phys. 155 (1959) 205. 